\title{
100th Anniversary of the Medical Faculty of Comenius University in Bratislava and its scientific journal "Bratislava Medical Journal"
}

In September 2018, the Faculty of Medicine at Comenius University in Bratislava begun its $100^{\text {th }}$ academic year. Thus, it is one of the oldest of the thirteen current faculties of the university, which was established by the law passed in June 1919 as the Czechoslovak State University in Bratislava. It was actually within the Faculty of Medicine that this new institution started an educational process in December 1919. By that time, it had already been renamed to Comenius University by a governmental decree. The educational process was supervised as well as carried out by a group of professors formerly from Charles University in Prague. One of them, Professor Kristián Hynek, a distinguished internal medicine expert, was appointed the first rector of the Comenius University. Therefore, the history of our alma mater has always been both historically and "genetically" intertwined with Charles University in Prague.

From the very beginning, the Faculty of Medicine of Comenius University (FM CU) played the role as an institution in charge of educating future physicians in the Slovakian context. Thanks to the previous academic experience, education and assistance of faculty founders, thousands of Slovak physicians, educators and scientists were educated here. They would later become leading authorities at their respective institutes and university facilities; thus, a continuation of successful educational and scientific activities has been secured. Hundreds of our alumnae and alumni have achieved an international recognition; let us mention especially Professor Jan Vilček, a distinguished microbiologist and immunologist.

One hundred years ago, professor Hynek introduced a Letter of Application to the president of the republic in person, which addressed the issue of improvement of the educational level in three points: 1 . foundation of a university, 2 . foundation of an association of Czechoslovak physicians (medical doctors), and 3. establishment of the Bratislava Medical Journal. The journal was established in 1921 and gave the opportunity to the teachers and the scientific workers to publish and thus broaden and preserve their experiences. Bratislava Medical Journal is the oldest scientific journal in the modern history of Slovakia. Professor Hynek was the first editorin-chief of the journal. The editorial preface to the first issue starts as follows: "Bratislava Medical Journal was established as an integral part of the building-up of the medical scientific life in Slovakia."

In the first decades after the journal's establishment, the content was published exclusively in Slovak and Czech language with abstracts in Russian, German, French or English language. This habit was in accordance with the official agenda of the newly founded FM CU to teach Slovak medical students in their native language, what turned out to be a substantial contribution to the development of science in Slovakia. Later, the papers were published with English abstracts, which was a sign of gradual transition toward bilingual version (abstract, results, figure captions, tables and conclusion were presented also in English). 
Bratislava Medical Journal has been published continuously from the foundation of the university and its medical faculty until present day. Despite sometimes uneasy conditions, under which the journal had to be published during its long history, Bratislava Medical Journal evolved into an internationally renowned and respected professional medical scientific journal. Since the beginning until today, the journal focused on the publishing of peer-reviewed papers mainly from the fields of clinical and experimental medicine, public health, novel therapies and practices, joint reports with scientific and postgradual focus, as well as papers about the history of medicine in Slovakia.

The arrival of information technologies, including a widespread access to internet, made it possible for the journal to present itself also in electronic version available on its own website (www.elis.sk). In 1991, as the first professional journal from Slovakia, Bratislava Medical Journal started to be indexed in internationally renowned databases. The journal is included in databases such as WoS, Medline, PubMed, Scopus, Thompson Scientific Publications Processing and more. The information databases feature also retrospectively included abstracts and full-text versions of papers published from 1965 until now. In 1993, the journal has become fully bilingual (Slovak-English) and since 1998 it has been defined as an international scientific journal published exclusively in English as Bratislava Medical Journal.

In 1950, when the academic activities were centered on the grounds of the Academy of Sciences, the publisher was changed and the editorial office was moved to the Academy of Sciences until 1993. This was the year when it was moved back to the grounds of the FM CU (the dean Professor Peter Mráz, in charge Assoc. Professor Marián Bernadič).

Many significant scientific personalities, who defined the directions of Slovak medicine and education, have been a part of the editorial board from the very beginning. The first mention rightfully belongs to Professor Ivan Hulin. Bratislava Medical Journal became an internationally highly recognized scientific journal under his leadership. Nowadays, the English version of the journal is a part of large and eminent international databases, its citations are SCI and it has the Impact factor of 0.86 . While until 1993 approximately 300 manuscripts were submitted annually, (ratio of published/rejected 1:1), now it is 1800 (ratio of published/rejected 1:12).

In 2012, Bratislava Medical Journal was declared a cultural and scientific heritage of the Slovak Republic by The Minister of Culture. We hope, at the occasion of its 100th Anniversary, the journal will be declared a cultural heritage of UNESCO.

Medical Faculty of Comenius University has contributed significantly to the development of general medical education also within a state-wide context. For instance, both The Faculty of Medicine at UPJŠ, Košice as well as the Jessenius' Faculty of Medicine, CU, in Martin had originally been founded as branches of the Faculty of Medicine, CU in Bratislava. Moreover, FM CU faculty has been participating in the educational process of Slovak Medical University, Bratislava until today. Additionally, the faculty boasts a significant share in respect to the yearly graduation rate of Slovak physicians: for instance, in 2017,325 out of 740 medical graduates studying in the Slovak language completed their education at the FM CU in Bratislava.

While the educational extent has been gradually growing, it was, unfortunately, at the expense of quality. For instance, the same premises that were available to educate 3000 pre-graduate students in the academic year 2014/2015 had previously served 1800 students in the academic year of 1999/2000. Research conditions worsened as well: activities of individual institutes were reduced and equipment 
remained rather outdated. For example, there is no operation room at the Institute of Anatomy anymore and the Institute of Histology lacked an electron microscope. New premises of basic science institutes have been expected to be built during the construction of a new university hospital (campus). A blind trust of the faculty bodies in promised rectifications, together with a clear absence of any real deeds, have brought the educational process to the very edge of a precipice.

Thus, the current FM CU management has been forced to emphasize various quality aspects as far as faculty activities are concerned. Firstly, we aim to provide appropriate conditions for both pre-graduate and postgraduate education. Therefore, at the very last moment, we managed to obtain the premises on Moskovská Street from the university management. After renovations, we moved a significant part of the educational process onto these premises and it has in turn enabled a revitalization of the deteriorated premises on Sasinkova Street. Currently, the first part of this process has nearly been completed. Not only the look of the whole facility is gradually changing, but several institutes have also been renovated. We have additionally supplemented much needed equipment as well as substantially improved the existing equipment of the Centre for Virtual and Simulation Education.

These measures have been taken while the faculty still remains significantly underfunded as far as the state budget is concerned. The analysis has shown that the state contribution covers only $42 \%$ of payroll funds; therefore, the faculty is able to economically survive only thanks to tuition of students currently enrolled in the English language program. Having first been a tool to enhance the faculty prestige, this concept has eventually become the means for the faculty's financial survival.

In many fields, the quality of education directly depends on the quality of research and scientific activities. We have thus analyzed the projects' outcomes as well as publication outputs of individual institutes and departments. This resulted in a compilation of the Main Research Areas which provides an actual performance overview in terms of individual facilities and also outlines a potential FM CU cooperation with Slovak and international institutions in the fields of neurosciences, cardiovascular, metabolic and endocrine diseases, and oncology.

We realize that a high-quality education requires an appropriate infrastructure and working conditions. Both faculty and students must simply work in a civilized, cultured and suitable environment; in an adequate material and technical setting as well as proper social background. Financial prospects must not fall into the lowest salary classifications, comparable even to those of unskilled laborers. Bearing all these challenges in mind, we started the current tenure, aiming at the payroll reform since the very beginning. December 2017 marked a historical milestone when, after negotiations among the FM CU, CU and the Ministry of Health of Slovakia, an amendment of university hospitals' deeds of foundation came into effect. This legislation clearly defines the status of the heads of the departments, health carerelated commitments of Faculty, status of faculty members working at the FM $\mathrm{CU}$ departments - who are paid approximately in the same manner at the moment as physicians who are employed by the hospitals. This development marked a major progress in respect to the faculty payroll policy, which aims at better wage conditions also for the faculty members of the Institutes (theoretical subjects) from the faculty's own resources. After introducing a new system of rewards and improving the already existing one, accompanying various achievements related to qualification increase, $\mathrm{PhD}$ research, student scientific conference ( $\breve{\text { VOOČ) }}$ involvement, etc., we have aimed to increase the motivation of both the faculty staff and students. 
Indeed, the expression "improving quality of performance" might sometimes sound rather empty. When trying to implement this concept in practice, a functional feedback between students and faculty is necessary. Therefore, this concept can be put into practice only via a thorough analysis of student questionnaires, meetings with students, Board of the Dean meetings, etc., followed by a feedback evaluation and a subsequent search for possible solutions, should such measures be required.

The most essential challenge for the upcoming years is our curriculum innovation. It will become necessary to innovate curriculum of all the subjects as well as to incorporate new methods into both basic science and clinical education. The one-way information flow during the lectures needs to be enhanced by more interactive techniques such as team-based learning, with an emphasis on their interdisciplinary content and the application of new IT technologies.

The direct interaction between a medical student and a patient represents the very foundation of practical education in clinical subjects. In the meantime, some of the facilities designated for these purposes have fallen into disrepair: minor refurbishments have not covered their actual needs and the equipment has remained rather poor. There is almost no single department that does not suffer from a lack of nurses or an insufficient number of physicians. The possibilities of clinical education are more limited when compared to the situation in the past. The faculty management will need to tackle the challenge of expanding options in the near future. It will be necessary to transfer some parts of the clinical education to the outpatient care. In addition, this education should be supplemented with new methods, such as Objective structured clinical examination (OCSE) using "actors", video recordings as well as live streaming from diagnostic and therapeutical procedures. With a substantial extension of virtual and simulation education (3D visualization, simulators, etc.), we can prepare our students for their first interaction with a patient.

Future graduates can become better prepared for the practice only thanks to an ever-improving interconnection between FM CU activities and teaching hospitals under the Ministry of health supervision, in which the final version of the University Hospital Statute and its implementation would represent the ideal case. To accept the status of the University hospital employee and the medical students should be the first step. Further essential conditions include appropriate selection criteria together with a transparent mechanism of appointing future heads of individual departments. Moreover a complex, modern university hospital is a necessary requirement. Should the new hospital fail to exist, the university health care would most probably cease to function, together with the whole system of medical education. This development would have an impact on the entire country of Slovakia.

As the Faculty of Medicine at Comenius University in Bratislava enters the second century of its existence, the faculty management is facing a challenging task. It will be necessary to make full use of faculty's personnel potential to defend this institution's position as the most distinguished medical faculty in Slovakia, renowned both at home and abroad. It is by no means possible to expect any quick changes, as our objectives can only be met when persisting in the path that has already been chosen. Only then the changes may become permanent. I believe we will be able to succeed in this respect and, as a reward, we shall be satisfied with the results of our effort. The meaningfulness of our vocation shall be reflected in the satisfaction of both our graduates and their future patients. 